\title{
The NMR Chemical Shift of Xenon-129 Dissolved in Polymers
}

\author{
J. B. Miller, J. H. Walton, ${ }^{1}$ and C. M. Roland \\ Naval Research Laboratory, Chemistry Division, Code 6120, Washington, D.C. 20375-5000 \\ Received December 28, 1992; Revised Manuscript Received June 7, $1993^{\circ}$
}

\begin{abstract}
The temperature dependence of the chemical shift of ${ }^{129} \mathrm{Xe}$ dissolved in polymers is examined in the context of the van der Waals interaction. It is found that above the glass transition temperature for the polymers studied herein, the ${ }^{129} \mathrm{Xe}$ chemical shift can be described by a 6-12 Lennard-Jones potential. The cell model of DiBenedetto is used to describe the average local environment of the amorphous polymer and sum over the pairwise interactions of the potential. We show the van der Waals shift model agrees reasonably well with the ${ }^{129} \mathrm{Xe}$ chemical shifts and provides the potential to determine solubility parameters and van der Waals radii of polymers.
\end{abstract}

\section{Introduction}

The ${ }^{129} \mathrm{Xe}$ chemical shift is particularly sensitive to the environment of the atom. Xenon is a noble gas and therefore interacts with its surroundings primarily through the van der Waals interaction. However, because xenon has a large number of electrons, even this small interaction produces a chemical shift relatively large in magnitude and with a strong temperature dependence. ${ }^{129} \mathrm{Xe}$ is $26 \%$ abundant and has a relatively large magnetogyric ratio, $\gamma$, so dilute amounts can be detected. For these reasons, ${ }^{129} \mathrm{Xe}$ NMR has been widely used to study solid materials including zeolites ${ }^{2}$ and clathrate compounds. ${ }^{3,4}$ More recently, the NMR of ${ }^{129} \mathrm{Xe}$ dissolved in polymers has been investigated..$^{5-10}$ In these studies, the ${ }^{129} \mathrm{Xe}$ chemical shift is generally correlated with a molecular parameter such as pore size or density through semiphenomenological theories which usually involve xenon-wall collision rates or the residence times of xenon atoms on the cavity walls. The NMR of xenon in liquids has also been used to test theories of solute-solvent interactions. . $^{11,12}$ We note that resonance shifts due to solute-solvent interactions arise from the physical interactions of the solute with its surroundings and are distinct from and additive with the more familiar chemical shifts which arise from the chemical structure of the solute. Xenon dissolved in polymers above the glass transition is in a more liquid-like environment; therefore it is appropriate to attribute the ${ }^{129} \mathrm{Xe}$ chemical shift to solute-solvent interactions.

We first briefly review the present understanding of the origin of resonance shifts due to solute-solvent interactions. In the van der Waals Shift Model section we introduce a new model of the van der Waals shifts applicable to polymers. We then describe in detail how the parameters necessary to implement the model are calculated in the Calculation of Parameters section. Finally, in the Results and Discussion section we present the results and discuss how a number of the assumptions made in the model affect the results.

The theory of resonance shifts due to solute-solvent interactions has received much attention over the years..$^{13}$ (Throughout this paper we will refer to the calculated values as resonance or van der Waals shifts and the measured values as chemical shifts.) The resonance shift of a solute molecule due to solute-solvent interactions is the sum of several contributions:

$$
\sigma=\sigma_{\mathrm{w}}+\sigma_{\mathrm{r}}+\sigma_{\mathrm{b}}+\sigma_{\mathrm{a}}+\sigma_{E}+\sigma_{E^{2}}
$$

The various contributions are defined as follows: $\sigma_{\mathrm{W}}$ is the

Abstract published in Advance ACS Abstracts, September 15, 1993. van der Waals dispersion interaction; $\sigma_{\mathrm{r}}$ is the repulsive interaction; $\sigma_{\mathrm{b}}$ is due to bulk susceptibility; $\sigma_{\mathrm{a}}$ arises from the magnetic anisotropy of the solvent molecules; $\sigma_{E}$ is due to the reaction field induced by the permanent electric dipole moment of the solute; and $\sigma_{E^{2}}$ is due to the permanent electric dipole moment of the solvent.

Rummens ${ }^{14,15}$ showed that for many polar solvents the $\sigma_{E^{2}}$ contribution is negligible. Since the local dipole moments in the polymers studied here are relatively weak, this contribution will be neglected. Xenon has no permanent dipole moment; therefore $\sigma_{E}$ is zero. A literature value for the bulk susceptibility of polyethylene of $\chi_{\mathrm{V}}=$ $1.01 \times 10^{-6}$ translates to a value for $\sigma_{\mathrm{b}}$ of about $2 \mathrm{ppm}$ for our geometry (a short cylinder with its axis perpendicular to the magnetic field) and is typical of hydrocarbons. The contribution of $\sigma_{\mathrm{a}}$ to the chemical shift of ${ }^{129} \mathrm{Xe}$ in organic solvents has been estimated to be ca. $1 \mathrm{ppm} .{ }^{11}$ These shifts are all negligible when compared to the $200 \mathrm{ppm}$ shifts typically observed for ${ }^{129} \mathrm{Xe}$ in polymers. Thus, the van der Waals dispersion and repulsive interactions are the dominant contributions to the ${ }^{129} \mathrm{Xe}$ chemical shift.

A number of theories have been advanced for the van der Waals shifts. ${ }^{13}$ One of two approaches is usually taken-either a statistical mechanical approach ${ }^{16,17}$ or a continuum model. ${ }^{18-21}$ In the statistical mechanical approaches the dispersive interaction between a pair of atoms or molecules is usually considered. The continuum approaches consider the solvent as a homogeneous medium. The van der Waals shift arises from polarization of the solute's electron cloud due to the reaction field 22 in the medium. ${ }^{23}$ The temperature dependence of chemical shifts has also been examined theoretically ${ }^{17}$ as well as experimentally in elemental phosphorus, ${ }^{24}$ methanol, ${ }^{25,26}$ solid ${ }^{27,28}$ and liquid ${ }^{27-29}{ }^{129} \mathrm{Xe},{ }^{129} \mathrm{Xe}$ in liquids, ${ }^{11,12}$ and ${ }^{129} \mathrm{Xe}$ in polymers ${ }^{7,10,30}$ among other systems. In the approaches appropriate to liquid solvents there is no restriction that the solvent and solute be different molecules. Below, we present a new model for the van der Waals shifts of solutes in amorphous polymers which takes into account the local structure in these materials.

\section{van der Waals Shift Model}

To model the van der Waals shift of ${ }^{129} \mathrm{Xe}$ in a polymer, we must be able to describe the van der Waals interaction between the xenon atom and the neighboring polymer chains. One approach previously taken has been to model the surrounding medium (polymer chains in our case) as a continuum. ${ }^{18-21}$ These models have met with some success describing the van der Waals shifts in liquids; however, they do not provide a good fit to our polymer 

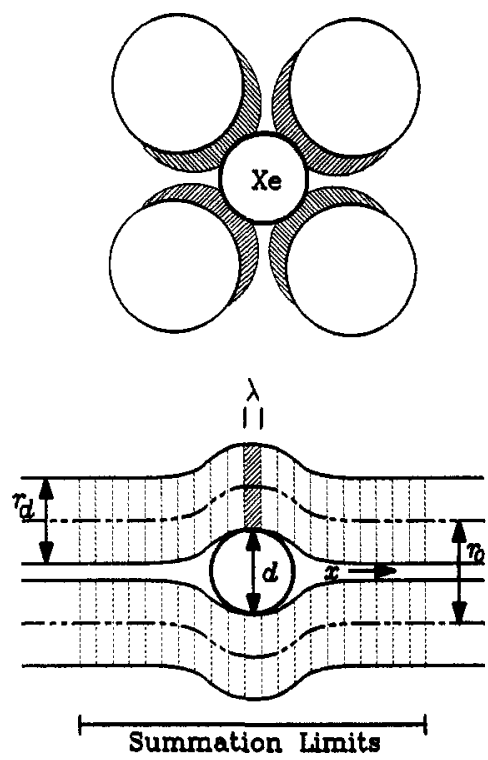

Figure 1. Schematic representation of xenon in a polymer matrix: the xenon is situated between four polymer chains arranged on a square lattice at the top; side view of xenon between two polymer chains at the bottom. The van der Waals diameter of the polymer chain is $r_{d}, r_{0}$ is the equilibrium spacing of the polymer chains, $\lambda$ is the spacing between centers on the chain, $d$ is the xenon diameter, and $x$ is the coordinate parallel to the polymer chains. $r *$ is approximated as $r_{d}$ at $T_{g}$. The DiBenedetto cell model accounts for the interaction between a center on one chain (shaded) and 21 centers on the opposite chain (summation limits).

data (presented below). This is not surprising considering that locally an amorphous polymer is anything but a homogeneous continuum. Statistical mechanical models, ${ }^{16,17}$ as they appear in the literature, are not applicable to solute molecules in polymers. Below, we present a model which specifically takes into account the local structure of amorphous polymers.

We will base our van der Waals shift model on a successful model of the diffusion of small molecules through an amorphous polymer developed by DiBenedetto ${ }^{31,32}$ and Pace and Datyner. ${ }^{33-35}$ The diffusion model describes the van der Waals interaction between a small molecule (xenon) and a polymer matrix, employing a Lennard-Jones 6-12 potential and a cell model to describe the local arrangement of the polymer chains. Below, we reproduce the important features of this model from the work of DiBenedetto and extend its application to NMR resonance shifts.

We begin with a description of the local structure of an amorphous polymer, following the cell model of DiBenedetto. $^{31}$ Although the global configuration of flexible chains is undeniably isotropic, the competition for space among chain segments confers locally some orientational order, with a persistence length on the order of a nanometer. ${ }^{31,36}$ We model the local arrangement as a bundle of parallel chains. A coordination number of 4 is assumed for the amorphous regions based on differences in density between crystalline and amorphous polymers (typically $10 \%$ ) and cohesive energy density calculations. ${ }^{33}$ Locally then, the polymer chains are modeled as smooth, cylindrical, parallel tubes arranged on a square lattice as illustrated in Figure 1. Each tube is assumed to be composed of $N$ equivalent centers separated by an average distance $\lambda$. Each center consists of a polymer chain backbone atom and all nonbackbone atoms bonded to it. Strictly speaking, the centers will not in general be equivalent; however, in the case of large diffusant molecules such as xenon, at any given instant all centers in the monomer will be in close proximity to the diffusant. Other minor variations are likely to be averaged by rapid translation of the diffusant. In the absence of a diffusant molecule the Lennard-Jones potential between one center and $2 j+1$ centers on a neighboring chain is

$$
\phi_{r}=\epsilon^{*} \sum_{i=-j}^{i=j}\left[\left(\frac{r^{*}}{r_{i}}\right)^{12}-2\left(\frac{r^{*}}{r_{i}}\right)^{6}\right]
$$

where $r_{i}$ is the equilibrium separation of the center in question from the $i$ th center on the neighboring chain and $r^{*}$ is the equilibrium chain separation in the low-temperature limit. (In the cited literature radial distances were denoted by $\rho$; we have switched to $r$ to eliminate confusion with the symbol for density, keeping the same system of subscripts and superscripts.) The average Lennard-Jones energy parameter ${ }^{33} \epsilon^{*}$ is given by

$$
\epsilon^{*}=\frac{\delta^{2} m^{*} \lambda}{3.88 \rho r^{*}}
$$

where $\delta$ is the polymer solubility parameter, $m^{*}$ is the average center molecular weight, and $\rho$ is the polymer density. For linear parallel chains $r_{i}$ can be written as

$$
r_{i}^{2}=r_{0}{ }^{2}+(i \lambda)^{2}
$$

where $r_{0}$ is the equilibrium separation between the two closest centers on neighboring chains, i.e., $i=0$. DiBenedetto has shown that for $j=10$, eq 2 can be approximated by ${ }^{31}$

$$
\phi_{r}=\frac{\epsilon^{*} r^{*}}{\lambda}\left[0.77\left(\frac{r^{*}}{r_{0}}\right)^{11}-2.32\left(\frac{r^{*}}{r_{0}}\right)^{5}\right]
$$

A diffusant molecule inserts into the interstitial space and, in the case of larger molecules such as xenon, bows the tubes outward, as indicated schematically in Figure 1. In the presence of a diffusant molecule, eq 5 becomes ${ }^{32}$

$$
\phi_{z}=\frac{\epsilon^{*} r^{*}}{\lambda}\left[0.77\left(\frac{r^{*}}{z}\right)^{11}-2.32\left(\frac{r^{*}}{z}\right)^{5}\right]
$$

where $z$ is the total chain separation due to the insertion of the diffusant molecule. Equation 6 presumes that the direct van der Waals interaction between the diffusant and polymer is negligible. We assume that the diffusant creates the hole it occupies in the polymer medium and is not limited to existing pockets of free volume large enough to accommodate it. This assumption is supported for polymers above $T_{\mathrm{g}}$ by the observations of Burgess $e t$ $a l .37$ on the transport of noble gases in polymers and by the NMR and dilatometry measurements of Sefcik et al. ${ }^{6}$ On the other hand, measurements of average free volume hole sizes ${ }^{38-40}$ and free volume hole size distributions ${ }^{41}$ in several polymers suggest that a large fraction of the free volume holes are large enough to accommodate a xenon atom. Above $T_{\mathrm{g}}$, however, the free volume holes are not static but fluctuate in volume and shape, assuming they are not associated with some form of static defect site. Static sites will make only a small contribution to the time-averaged van der Waals shift unless they exist in large numbers because of the rapid diffusion of xenon through the polymers: $1.4 \times 10^{-7} \mathrm{~cm}^{2} / \mathrm{s}$ in polyisoprene (natural rubber) at $298 \mathrm{~K} .42$ The combination of rapid xenon diffusion and free volume fluctuation will result in the xenon experiencing an average free volume environment closely resembling that described above.

DiBenedetto equates $E_{\mathrm{d}}$, the energy barrier to diffusion, with $N_{\mathrm{a}} \Delta \phi_{\mathrm{T}}$, where $N_{\mathrm{a}}$ is Avogadro's number and $\Delta \phi_{\mathrm{T}}$ is the total increase in potential energy for inserting the 
diffusant: ${ }^{32}$

$$
\Delta \phi_{\mathrm{T}}=\int_{-\infty}^{+\infty}\left(\phi_{z}-\phi_{r}\right) \mathrm{d} x
$$

The integration axis, $x$, is parallel to the polymer chains. During the diffusion process it is assumed that the chains separate by a constant distance $z$ for some number of centers $n$, which is assumed to be a function of polymer chain flexibility only, and then return to their equilibrium spacing; therefore

$$
E_{\mathrm{d}}=N_{\mathrm{a}} n\left(\phi_{z}-\phi_{\mathrm{r}}\right)
$$

A value for $n$ can be obtained from $E_{\mathrm{d}}$ data for diffusants of varying diameter and eq 8 . In their version of the model, Pace and Datyner ${ }^{33}$ account for the dependence of $z$ on $x$ in eq 7 through a chain-bending modulus parameter. They note that $\Delta \phi_{\mathrm{T}}$ is essentially a linear function of temperature over ranges of ca. $50 \mathrm{~K}$ above $T_{\mathrm{g}}$ and equate $E_{\mathrm{d}}$ with the apparent activation energy:

$$
\Delta E_{\mathrm{app}}=\Delta \phi_{0}-\frac{\delta\left(\Delta \phi_{\mathrm{T}}\right)}{\delta T} T_{0}
$$

where $\Delta \phi_{0}$ equals $\Delta \phi_{\mathrm{T}}$ at the reference temperature $T_{0}$.

In the following discussion we follow the DiBenedetto model. We note xenon is a large atom, so that chain bending around the atom will occur over a large radius of curvature. The polymers studied herein have flexible backbones; therefore we assume that they can follow the curvature of the xenon atom. We then write the total increase in potential energy as

$$
\Delta \phi_{\mathrm{T}}=n\left(\phi_{z}-\phi_{r}\right)
$$

We assume that the separation of the polymer chains, relevant to the ${ }^{129} \mathrm{Xe}$ chemical shift, extends only over the diameter of the xenon atom; we thus approximate $n$ as $d / \lambda$. In the Results and Discussion section we discuss some of the qualitative implications of these assumptions.

In an early continuum model Linder derives an equation for the van der Waals shift of a solute molecule in a solvent: ${ }^{19}$

$$
\sigma_{\mathrm{w}}=C F
$$

$C$ is composed of a scaling constant and several molecular parameters of the solute, and $F$ is the dispersion free energy of the solute. $F$ may also be associated with the work required to create a cavity with dimensions of the solute in the dielectric continuum. ${ }^{18}$ In analogy to the Linder continuum model, $\Delta \phi_{\mathrm{T}}$ is the difference in the energy of the polymer chain configuration with and without the xenon atom; therefore, we write

$$
\sigma_{\mathrm{w}}=K \Delta \phi_{\mathrm{T}}
$$

for a solute molecule in an amorphous polymer. Equation 12 is the fundamental relationship extending the DiBenedetto cell model to predict van der Waals shifts.

The left-hand side of eq 12 should be $\sigma_{\mathrm{w}}+\sigma_{\mathrm{z}}$ because $\Delta \phi_{\mathrm{T}}$ includes both dispersive and repulsive terms. For simplicity, we will refer to this shift as $\sigma_{\mathrm{w}}$ only throughout the remainder of the text. For conformity with the literature on van der Waals shifts we have referred to the resonance shifts in the $\sigma$ scale; however, we report the data in the more familiar $\delta$ scale: $\delta=-\sigma$.

It is not clear from the cited literature and our van der Waals shift model whether the scaling constant $K$ is determined by the properties of the solute alone or of the solute and solvent combined. The scaling constant describes how the potential due to polymer chains distorts the electron cloud about the xenon, giving rise to the van $a$
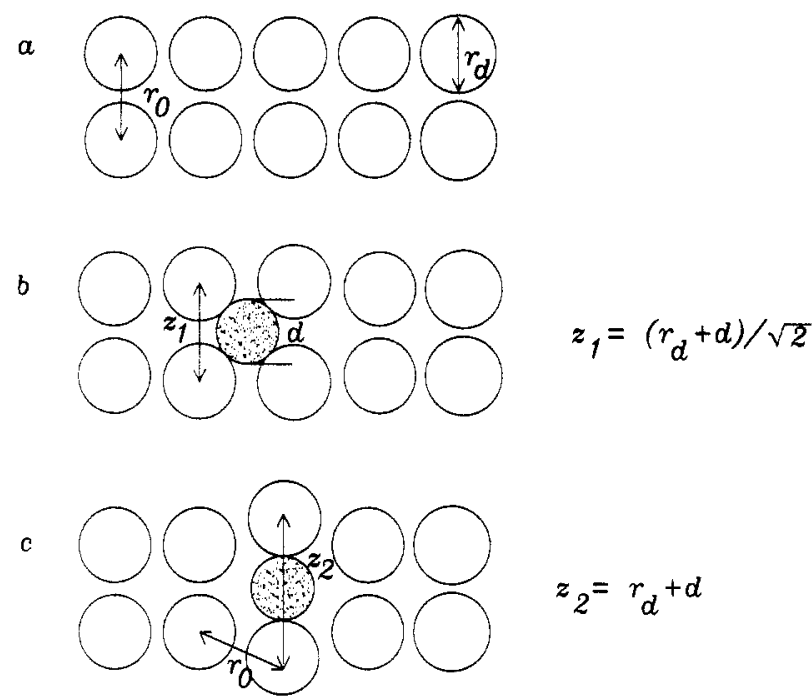

Figure 2. (a) Polymer chains on a square lattice viewed end on. (b) The polymer chains are displaced by a xenon atom in site one. (c) The polymer chains are displaced by a xenon atom in site two. It is assumed that only chains in direct contact with the xenon atom are perturbed by its presence. All other chains rearrange to maintain their equilibrium spacing. The definitions of the variables are given in the text.

der Waals shift, $\sigma_{w}$. Although in analogy to the scaling constant of eq $11 \mathrm{~K}$ may be calculated, ${ }^{43}$ we take it to be the only adjustable parameter. For the present analysis we assume that $K$ of eq 12 is determined by the properties of the solute only, therefore having the same value for all polymers.

\section{Calculation of Parameters}

To evaluate eq 12 , we must determine the values of several parameters including $r^{*}, r_{0}, \epsilon^{*}$, and $z$. We will also consider the temperature dependence of these terms. We start by determining the functional form of $z$ and leave the consideration of its temperature dependence until later in this section.

The first site into which a diffusant molecule can insert is in the interstitial space between four polymer chains as shown in Figure 2b. Four interchain spacings are affected by the diffusant; we assume that polymer chains not in direct contact with the diffusant rearrange to maintain their equilibrium spacing. Under the assumption that the chains separate symmetrically, $z$ is given by

$$
z_{1}=\left(r_{d}+d\right) / 2^{1 / 2}
$$

where $d$ is the diameter of the diffusant molecule $(0.49$ $\mathrm{nm}^{34}$ for xenon) and $r_{d}$ is the effective polymer diameter with which the diffusant interacts.

Pace and Datyner ${ }^{33}$ used $r^{*}$ for $r_{d}$, implying that the diffusant molecule interacts with the local free volume and increases the chain separation by only the difference between the diffusant diameter and the local free volume. The alternative, using $r_{0}$ for $r_{d}$, where the diffusant does not interact with the local free volume causes a large increase in the van der Waals energy. We will also assume that the diffusant interacts with the local free volume, although we will treat $r_{d}$ as temperature dependent as discussed below.

Since the polymer chains are isotropically configured, xenon must move perpendicular to them as well as parallel. During a jump perpendicular to the chains, the xenon will push the polymer chains farther apart, as shown in Figure 2c. In this second site, six polymer chains surround the diffusant but only one nonequilibrium interchain spacing 
results. For the two chains in contact with the diffusant, $z$ is given by

$$
z_{2}=r_{\mathrm{d}}+d
$$

Again we assume that all other polymer chains rearrange to maintain their equilibrium spacing.

In the simple cell model of amorphous polymers we identified two distinct sites into which a xenon atom can insert. During the course of diffusion, the xenon encounters both sites. In the fast diffusion limit the xenon will move rapidly between sites. We use the NMR definition of fast diffusion: the xenon must diffuse through regions of heterogeneous microstructure such that a true average of the polymer microstructure is sampled in a time short compared to the NMR time scale, in this case milliseconds. The ${ }^{129} \mathrm{Xe}$ resonance shift reflects the time-average potential the xenon experiences during diffusion. A single narrow peak should result in the NMR spectrum, which is the case for the polymers studied here at temperatures above $T_{\mathbf{g}}$.

To calculate the ${ }^{129} \mathrm{Xe}$ resonance shift, we need to know the relative amounts of time a xenon atom spends in each site. The relative times spent in each site can be determined, in principle, from the Boltzmann populations of the sites, given the potential associated with each site. For a typical polymer, the calculated values of the potentials are similar for both sites, site one having the higher potential in most cases. However, the calculated values of the potentials depend strongly on the value of $n$ (eq 10) we choose, introducing a large uncertainty in the values of the potentials. (Fortunately, the ${ }^{129} \mathrm{Xe}$ resonance shifts we calculate are independent of $n$; any error in $n$ is compensated by our choice of $K$ in eq 12.) Furthermore, in our approximation, $n$ is the same for both sites. Site one requires a smaller chain separation than site two to accommodate a xenon atom, suggesting the possibility of a slightly smaller value of $n$ for site one. This would tend to make the potentials for the two sites more similar. In light of the uncertainty in the value of $n$, we will treat the potentials of the two sites as equal; therefore the ${ }^{129} \mathrm{Xe}$ resonance shift will reflect an average of the contributions from sites one and two. The implications of assuming equal potentials are addressed below.

The polymer chains are approximated as smooth tubes in our van der Waals shift model, and the free volume is just the volume between the tubes. The smooth tube approximation is not appropriate for polymers with bulky side groups. In particular, additional free volume should be available between the side groups projecting from the tubes. The amount of additional free volume cannot easily be quantified, and not all of it may be available to large diffusant molecules. Pace and Datyner ${ }^{35}$ employed a diffusant diameter reduction factor to account for additional free volume from large side groups on polymers, and we will follow this approach.

We are interested in the temperature dependence of $\sigma_{\mathrm{w}}$ which reflects the temperature dependence of $r_{0}$ and $r_{d}$. The polymer chains are arranged on a square lattice in our van der Waals shift model and we assume that the polymer fills space; therefore the volume of one center on a chain, including free volume, is

$$
V_{\mathrm{c}}=r_{0}^{2} \lambda=\frac{m^{*}}{\rho N_{\mathrm{a}}}
$$

where $N_{\mathrm{a}}$ is Avogadro's number. The temperature de-
Table I. Parameters Used To Calculate ${ }^{129}$ Xe van der Waals

\begin{tabular}{|c|c|c|c|c|c|c|c|c|}
\hline & $\begin{array}{c}\delta^{\delta^{2}} \\
\left(\mathrm{~J} / \mathrm{cm}^{3}\right)^{a}\end{array}$ & $\begin{array}{c}T_{0} \\
(\mathbf{K})^{?}\end{array}$ & $\begin{array}{c}\rho_{0} \\
\left(\mathrm{~g} / \mathrm{cm}^{3}\right)^{?}\end{array}$ & $\begin{array}{l}\alpha \times 10^{4} \\
(1 / \mathrm{K})^{?}\end{array}$ & $\underset{(\mathrm{K})_{b}}{T_{5}}$ & $f_{g} ?$ & $\underset{(\mathrm{g} / \mathrm{mol})}{\mathrm{m}^{*}}$ & $\begin{array}{c}\lambda \\
(\mathbf{n m})^{?}\end{array}$ \\
\hline & & & & 6.4 & 201 & 0.026 & 17.0 & 0.102 \\
\hline & & & & & & & & $0.11 f$ \\
\hline & & $298^{h}$ & $1.23^{?}$ & 6.0 & $239^{i}$ & $0.025 j$ & & $0.10^{k}$ \\
\hline & & $298^{?}$ & & & & & & $0.10^{?}$ \\
\hline & 2 & 298 & 0.915 & 5.4 & 5 & 0.02 & & 0.116 \\
\hline & & 29 & & 8. & & 0.0 & & 0.15 \\
\hline & & & & & & 0.02 & & 0.10 \\
\hline & & 348 & .1 & $3.8(2.4)^{t}$ & 335 & 0.025 & 57.0 & 0.105 \\
\hline
\end{tabular}
Shifts

${ }^{a}$ Reference 34. ${ }^{b}$ Reference $44 .{ }^{c}$ Reference $49 .{ }^{d}$ Reference 45. e Reference 47. $f$ By analogy to polypropylene. 8 Reference $46 .{ }^{h}$ Reference $31 .{ }^{i}$ Reference $48 . j$ General value for polymers. ${ }^{k}$ By analogy to polyisoprene. ${ }^{l}$ Thermal expansion coefficient above $T_{z}$; thermal expansion coefficient below $T_{B}$ in parentheses.

pendence of the volume can be well approximated by

$$
V_{c}(T)=\frac{m^{*}}{\rho_{0} N_{\mathrm{a}}}\left(1+\alpha\left(T-T_{0}\right)\right)
$$

where $\alpha$ is the thermal volume expansion coefficient taking into account both the expansion of the chain diameter and the increase in free volume, and $\rho_{0}$ is the density at the arbitrary reference temperature $T_{0}$. Volume thermal expansion above $T_{g}$ reflects the temperature dependence of the chain configuration, which is dominated by the bond rotational potentials. ${ }^{36}$ Thermal expansion will occur predominantly perpendicular to the chain axis, whereby the temperature dependence of $\lambda$ over the temperature ranges in this study can be neglected, yielding

$$
r_{0}(T)=\left(\frac{m^{*}}{\rho_{0} \lambda N_{\mathrm{a}}}\left(1+\alpha\left(T-T_{0}\right)\right)\right)^{1 / 2}
$$

We calculate $r^{*}$ from $V_{c}^{*}$, the volume of a center in the low-temperature limit, by eq 15 . Ignoring thermal expansion below the glass transition temperature, $T_{\mathrm{g}}$, we approximate $V_{c}^{*}$ as the difference between the total volume and free volume at $T_{\mathrm{g}}$. Using the ratios $f_{\mathrm{g}} \equiv V_{\mathrm{f}}\left(T_{\mathrm{g}}\right) / V\left(T_{\mathrm{g}}\right)$ from the literature, ${ }^{44}$ we may write $r^{*}$ as

$$
r^{*}=\left(\frac{m^{*}}{\rho_{0} \lambda N_{\mathrm{a}}}\left(1+\alpha\left(T_{\mathrm{g}}-T_{0}\right)\right)\left(1-f_{\mathrm{g}}\right)\right)^{1 / 2}
$$

We note that our method of estimating $r *$ sets it equal to $r_{d}$, the van der Waals chain diameter at $T_{\mathrm{g}}$, resulting in $r_{\mathrm{d}}$ being necessarily less than $r^{*}$ below $T_{\mathrm{g}}$. This is not an unrealistic result: the minimum in eq 5 occurs when $r_{0}=$ $0.83 r^{*}$, predicting that both $r_{0}$ and $r_{\mathrm{d}}$ can be less than $r^{*}$. We calculate $r_{d}$ from eq 18 by substituting $T$ for $T_{\mathrm{g}}$ and $\alpha_{0}$, the thermal expansion coefficient of the chains excluding free volume, for $\alpha$. We assume a value for $\alpha_{0}$ of $2.2 \times 10^{-4} \mathrm{~K}^{-1}$, typical for many polymers, for all polymers studied here.

The solubility parameter, $\delta$, is defined as

$$
\delta=\left(\Delta E^{\mathrm{v}} / V_{\mathrm{m}}\right)^{1 / 2}
$$

where $\Delta E^{v}$ is the energy of vaporization and $V_{m}$ is the molar volume. Since $V_{\mathrm{m}}$ is given by $\mathrm{m}^{*} / \rho$, then by eq 19 $\delta^{2}$ has the temperature dependence of $\rho$, whereby $\epsilon^{*}$ is temperature independent (eq 3 ). It is important that $\delta^{2}$ and $\rho$ are determined at the same temperature; we will use values of $\delta^{2}$ and $\rho$ at $298 \mathrm{~K}$ in subsequent calculations.

Other parameters needed to calculate $\sigma_{\mathrm{w}}$ are available from the literature and are listed in Table I. A diffusant diameter reduction factor will be applied to the data of poly(vinylethylene) (PVE) and poly(ethyl methacrylate) (PEMA), the only two polymers studied with large side groups. A value of the diffusant diameter reduction factor 


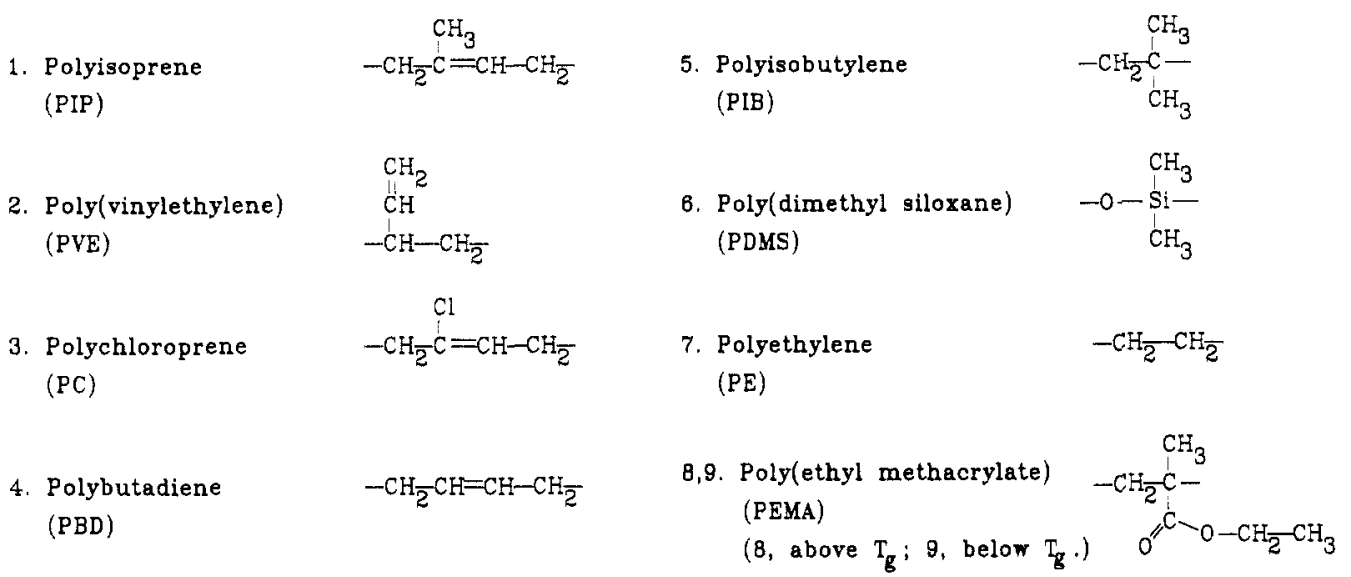

Figure 3. Polymers studied herein and their stuctures. The numbers in this figure correspond to the numbers in Figures 4 and 5.

for PVE is not available from Pace and Datyner; by comparing values of $r_{0}$ with other polymers with side groups, ${ }^{35}$ particularly polypropylene, we estimate a diameter reduction factor of $0.05 \mathrm{~nm}$ for PVE. From Pace and Datyner, ${ }^{35}$ the diffusant diameter reduction factor for the PEMA ranges from 0.14 to $0.16 \mathrm{~nm}$. We note however that our value of $r^{*}, 0.892 \mathrm{~nm}$, is smaller than their reported value of $0.918 \mathrm{~nm} .{ }^{34}$ Furthermore, a value of $0.14 \mathrm{~nm}$ leaves enough free volume in site one to accommodate a xenon atom, thereby making the potential zero in our model. We will therefore use a value of 0.10 $\mathrm{nm}$ for the PEMA diffusant diameter reduction factor.

\section{Experimental Section}

Polymer samples were sealed in a xenon atmosphere at ca. 10 atm as previously described. ${ }^{10}$ The samples were allowed to thermally equilibrate in the NMR probe for at least 30 min before data acquisition. Sample temperatures were measured with a thermocouple proximate to the sample; temperature stability was greater than $\pm 0.2 \mathrm{~K}$ over the course of data acquisition.

NMR measurements were made at the ${ }^{129} \mathrm{Xe}$ frequency of 83 $\mathrm{MHz}$; free induction decays (fids) were generated with a $30^{\circ} \mathrm{rf}$ pulse with a high repetition rate to maximize the signal-to-noise ratio in a minimum amount of time. The reported chemical shifts are relative to gas at 0.5 atm and are precise to $\pm 0.2 \mathrm{ppm}$. No attempt was made to correct for the bulk susceptibility.

The seven polymers were studied in one of two macroscopic physical blends, except for the polyethylene (PE). Physically blending the polymers allowed us to measure the chemical shifts of several polymers simultaneously. One blend consisted of polyisobutylene (PIB), polychloroprene (PC), and cis-1,4-polyisoprene (natural rubber, PIP). The other blend consisted of 1,4-polybutadiene (PBD), poly(vinylethylene) (97\% 1,2-polybutadiene, PVE), and poly(dimethylsiloxane) (PDMS). All were of high $\left(>10^{5}\right)$ molecular weight. We also report data for poly(ethyl methacrylate) (PEMA), obtained by Professor T. Stengle (see ref 7). The structures of these polymers are shown in Figure 3.

\section{Results and Discussion}

In order for ${ }^{129} \mathrm{Xe}$ NMR to provide useful information on polymers, the xenon must not change the properties of the polymer. Two pieces of evidence suggest this is the case. First, the xenon loading is low. Qualitatively comparing the respective signal intensities from ${ }^{129} \mathrm{Xe}$ dissolved in the polymer and in the surrounding volume (gas phase) indicates roughly a molar ratio 0.02 xenon to polymer repeat unit. ${ }^{129} \mathrm{Xe}$ NMR measurements on these same polymers at pressures lower than 10 atm indicate that the signal intensity increases with pressure but the chemical shift is constant; $; 0$ therefore, $\mathrm{Xe}-\mathrm{Xe}$ collisions are rare events and do not contribute to the chemical shift at these concentrations. Second, phase transitions in

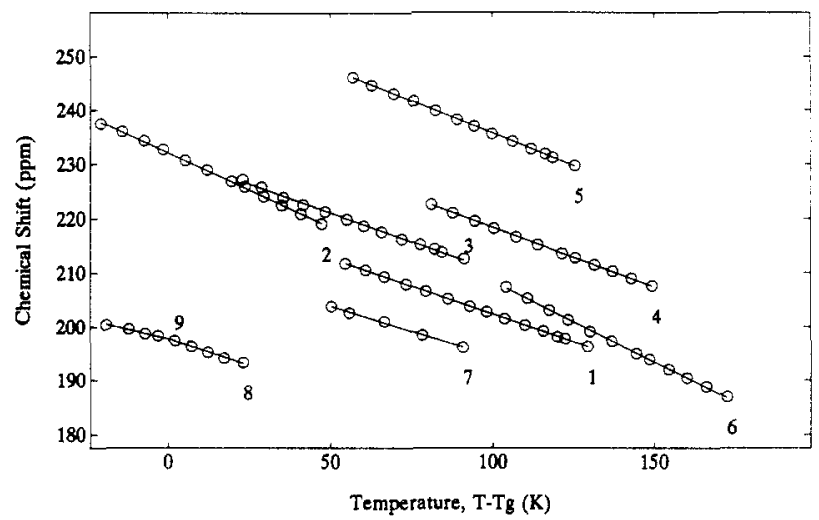

Figure 4. Plots of the ${ }^{129} \mathrm{Xe}$ chemical shifts, $-\sigma$, as a function of temperature for eight polymers. The numbers on the plot refer to the polymers as listed in Figure 3. The temperature coordinate, $T-T_{\mathrm{g}}$, is referenced to $T_{\mathrm{g}}$ in order to disperse the data. The linearity of the data can be observed in the plot.

polymers, as observed by their effect on the ${ }^{129} \mathrm{Xe}$ NMR spectrum, occur at the same temperatures as observed by other techniques. At temperatures just above the $T_{\mathrm{g}}$ of neat PVE, the ${ }^{129} \mathrm{Xe}$ line width is observed to increase for our PVE sample (the only polymer studied near its $T_{\mathrm{g}}$ ) and continues to increase as the temperature is decreased further, suggesting negligible plasticization by the xenon. The observed lower critical solution temperature for a blend of low molecular weight PIP and PBD by ${ }^{129} \mathrm{Xe}$ NMR is at the expected temperature. ${ }^{50}$ Stengle and Williamson ${ }^{7}$ also observe the glass transition in PEMA to occur at the expected temperature with ${ }^{129} \mathrm{Xe} \mathrm{NMR.}$

The chemical shift of ${ }^{129} \mathrm{Xe}$ dissolved in these polymers is a linear function of temperature for the temperature ranges used here, as shown in Figure 4. (The data are plotted on a temperature scale relative to the respective glass transition temperatures.) We will therefore compare the experimental chemical shifts and our van der Waals shift model's predictions in terms of a slope, $\mathrm{d}\left(-\sigma_{\mathrm{w}}\right) / \mathrm{d} T$, and intercept, the resonance shift, $-\sigma_{\mathrm{w}}$, at $273 \mathrm{~K}$ for each polymer. Comparison of the experimental and predicted slopes indicates how well we can reproduce the temperature dependence of the chemical shifts, whereas agreement of the intercepts indicates the model's ability to predict the magnitudes of the chemical shifts. In Figure 5A we plot the slope calculated from our model versus the slope measured from chemical shift data, and in Figure 5B we plot the calculated intercept versus the measured intercept. For clarity the points of the plots have been numbered; the numbers may be related to the polymers through Figure 3 . Lines having slopes of 1 and passing through the origin are provided as visual aids; they are not fits to the data. 

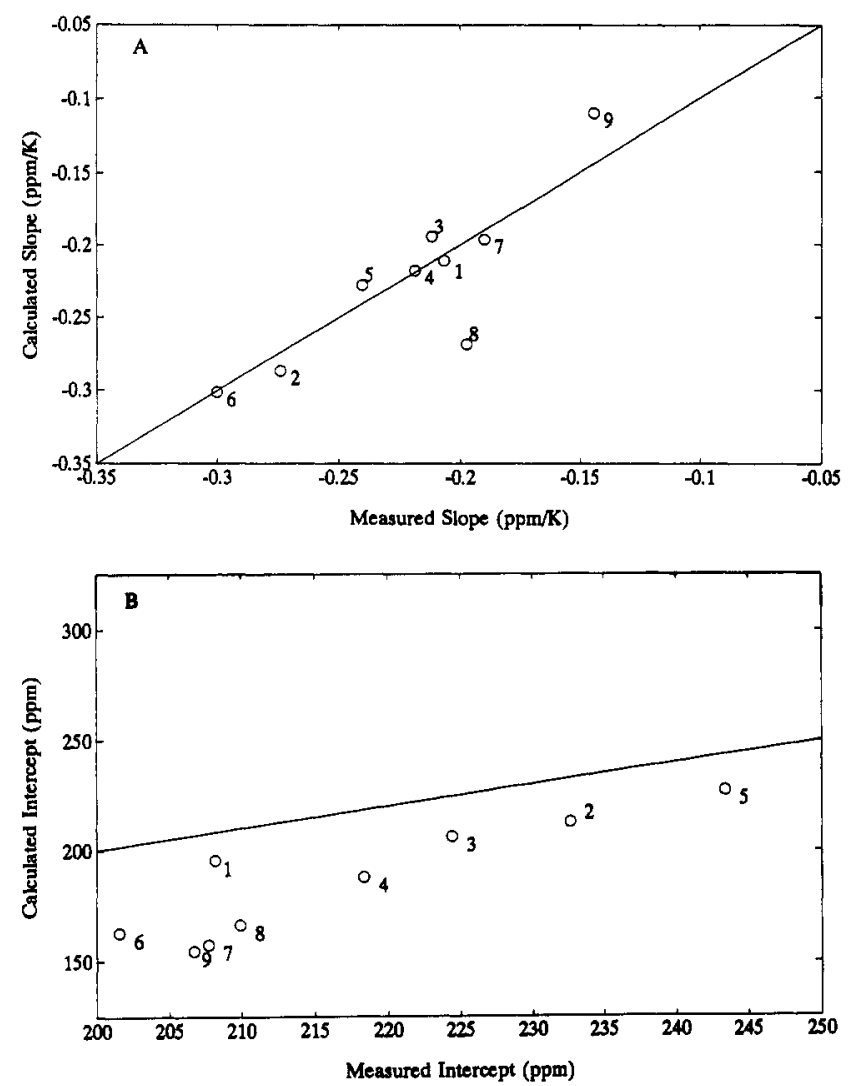

Figure 5. (A) Plot of the predicted slope, $d\left(-\sigma_{w}\right) / d T$, from eq 12 versus the slope, $\mathrm{d}(-\sigma) / \mathrm{d} T$, from the chemical shift data plotted in Figure 4. (B) Plot of the predicted intercept from eq 12,- $\sigma_{\mathrm{w}}$ at $273 \mathrm{~K}$, versus the chemical shift, $-\sigma$, at $273 \mathrm{~K}$ extrapolated from the data of Figure 4. It is assumed that the xenon occupies the local free volume and that the fast exchange limit between the two sites applies as discussed in the text.

As can be seen from Figure 5A, our van der Waals shift model predicts the temperature dependence of the ${ }^{129} \mathrm{Xe}$ chemical shift to within a few percent, with the exception of PEMA below $T_{\mathrm{g}}$. These results are quite good considering the eschewal of adjustable parameters, other than $K$. Moreover, some quantities used in the calculations are of limited or unknown accuracy, particularly the thermal expansion coefficient and the solubility parameter, for which a range of values appears in the literature (see Table II). The only adjustable parameter, $K, 0.012 \mathrm{~J}^{-1}$, an average value applicable to all polymers, was determined by minimizing the differences between the calculated and measured slopes (Figure 5A) for polymers 1-7.

This simple model predicts the magnitude of the resonance shift less accurately than the temperature dependence; all of the calculated values are low with differences in the range of $10-25 \%$ for most polymers (Figure 5B). (Discrepancies between the predicted resonance shifts and the measured chemical shifts due to other contributions such as bulk susceptibility will be only of the order of 1-2\%.) However, the van der Waals shift model has a sixth-power dependence on the polymer chain diameter (including free volume), $r_{0}$, and the van der Waals chain diameter in the low-temperature limit, $r *$. Hence, as with the temperature dependence, the relatively large differences between the predicted and measured resonance shifts may arise from small errors in the various quantities used to calculate $r_{0}$ and $r^{*}$.

To give some indication of the rather small magnitude of the errors in the polymer parameters $\left(\alpha, \rho_{0}, f_{\mathrm{g}}, \lambda\right.$, etc. $)$ necessary to account for the discrepancies in Figure 5 , we have calculated the values of the square of the solubility parameter, $\delta^{2}$, and $r^{*}$ required to exactly fit the model to
Table II. Comparison of Literature Values of Parameters and Values Obtained from Best Fit of the Model to the Data

\begin{tabular}{|c|c|c|c|c|c|c|}
\hline & $\underset{\left(\mathrm{J} / \mathrm{cm}^{3}\right)^{a}}{\delta_{\text {lit. }}}$ & $\begin{array}{c}\delta^{2} \mathrm{fit} \\
\left(\mathrm{J} / \mathrm{cm}^{3}\right)^{b}\end{array}$ & $\begin{array}{c}\Delta \delta^{2}= \\
\left(\delta^{2} \text { fit }\right. \\
\left.\delta^{2}{ }_{\text {lit. }}\right) / \delta^{2}{ }_{\text {lit. }} .\end{array}$ & $\begin{array}{l}r_{\text {calc }}^{*} \\
(\mathrm{~nm})\end{array}$ & $\begin{array}{l}r^{*} \text { fit } \\
(\mathrm{nm})\end{array}$ & $\begin{array}{c}\Delta r^{*}= \\
\left(r^{*} \text { fit }^{-}\right. \\
\left.r^{*} \text { calc }^{2}\right) / r_{\text {calc }}^{*}\end{array}$ \\
\hline $\begin{array}{l}\text { PIP } \\
\text { (NR) }\end{array}$ & $261-272$ & 263 & -0.034 & 0.524 & 0.531 & 0.012 \\
\hline PVE & 275 & 272 & -0.010 & 0.661 & 0.669 & 0.012 \\
\hline $\mathrm{PC}$ & $280-358$ & 286 & 0.023 & 0.529 & 0.528 & -0.002 \\
\hline PBD & $295-310$ & 311 & 0.014 & 0.471 & 0.482 & 0.024 \\
\hline PIB & $260-271$ & 260 & 0.000 & 0.637 & 0.638 & 0.002 \\
\hline PDMS & $223-230$ & 226 & -0.018 & 0.577 & 0.603 & 0.044 \\
\hline & $261-268$ & 306 & 0.143 & 0.445 & 0.462 & 0.038 \\
\hline PEMA $^{c}$ & $302-516$ & 340 & 0.018 & 0.892 & 0.913 & 0.024 \\
\hline PEMA $^{d}$ & $302-516$ & 416 & 0.244 & 0.892 & 0.892 & 0.000 \\
\hline
\end{tabular}

a Values taken from refs 31 and 34 and the observed values of ref 46. ${ }^{b}$ Based on an adjusted value of $K=0.011 \mathrm{~J}^{-1}$. ${ }^{c}$ From data above $T_{\mathrm{g}}$. ' From data below $T_{\mathrm{g}}$.

the data. We chose $r^{*}$ as a variable because its calculation involves all of the polymer parameters except for $\delta$. We also adjust the value of $K$ to keep changes in $\delta^{2}$ centered about zero. The results are listed in Table II along with the fractional changes in these parameters $\left(\Delta \delta^{2}=\left(\delta^{2}\right.\right.$ fit $\left.\delta^{2}{ }_{\text {lit. }}\right) / \delta^{2}{ }_{\text {lit. }}$ and $\left.\Delta r^{*}=\left(r^{*}{ }_{\text {int }}-r_{\text {calc }}^{*}\right) / r_{\text {calc }}^{*}\right)$. The fractional changes required for perfect agreement with our data are small, a few percent in most cases; the new value of $K$ is $0.011 \mathrm{~J}^{-1}$. The largest changes in $r^{*}$ occur for PDMS and $\mathrm{PE}$, which had the largest differences between the calculated and measured intercepts. The fit value of $r^{*}$ for $\mathrm{PE}, 0.462 \mathrm{~nm}$, is close to the value of $0.470 \mathrm{~nm}$ estimated from heats of vaporization by Brandt ${ }^{51}$ as well as the value of $0.463 \mathrm{~nm}$ obtained by Pace and Datyner. ${ }^{34}$ Large changes in $\delta^{2}$ for PE and PEMA below $T_{\mathrm{g}}$ are also observed. We note that the value of $\lambda$ for $P E$ is some $20 \%$ larger than for the other polymers with carbon backbones; errors in $\lambda$ will appear as errors in $\delta^{2}$ in this fit.

It may seem surprising that the resonance shifts predicted by the van der Waals shift model agree with the measured chemical shifts as well as they do, considering the simplicity of the model and some of the assumptions made. The model presents an ideal picture of polymer chains aligned on a square lattice with a stationary xenon atom between the chains. In reality there are local fluctuations in the polymer chain packing density and orientation. The fact that the model fits the data so well indicates that the average environment in the polymer above $T_{\mathrm{g}}$ has properties close to those described by the model. Several qualitative arguments may explain why this simple square lattice model is successful in describing the average environment in the polymer above $T_{\mathrm{g}}$.

First, the model's requirements for chain alignment are probably somewhat stricter than necessary. The cartoon lattices of Figure 2 depict parallel chains on a square lattice, which is convenient for picturing diffusion mechanisms. The lattice can also be viewed as parallel, horizontal planes of parallel chains, the chains in adjacent planes being parallel to each other. However, the summation of pairwise interactions between a center on one chain and the centers on an adjacent chain (eqs 2 and 5) does not require the two chains to be parallel. Furthermore, our calculations of $z$ (eqs 13 and 14) do not require the chains in adjacent planes to be parallel either, although this muddles the picture of the diffusion process somewhat.

Second, although the choice of a particular lattice, square in the present case, is necessary to determine the values of $z$, we expect other lattice structures to give similar results. We have calculated $z$ for the hexagonally close packed lattice and find that, with a small change in $K$, the 
calculated slopes and intercept differ from the square lattice model by only ca. $2 \%$ and $10 \%$, respectively. Surprisingly, the predicted intercepts are smaller for the hexagonally close packed lattice than for the square lattice. The potential of site two is essentially the same for both lattices since $z_{2}$ will be the same for all lattices and disruptions to neighboring chains will make at most only a minor contribution (zero in the square lattice model). In the hexagonally close packed lattice $z_{1}$ is somewhat larger than in the square lattice because of closer chain packing but the total potential of site one will be smaller because only three polymer chains are affected by the presence of the diffusant in the hexagonally close packed lattice as opposed to four in the square lattice. Presumably for more than four chains surrounding the diffusant site, the potential of site one will be slightly larger than in the square lattice due to the larger number of chains affected by the presence of the diffusant. The square lattice model therefore gives good average values for the two potentials.

Third, the ${ }^{129} \mathrm{Xe}$ NMR spectrum from each of these polymers consists of a single narrow resonance indicating that the xenon is diffusing rapidly on the NMR time scale of milliseconds. The spectrum therefore reflects an average of the environments formed by local fluctuations in density. For small fluctuations the van der Waals potentials will vary linearly with density, as is clearly seen in the linear variation of the van der Waals shift with temperature. The average of the potentials (both sites) over the local densities is therefore equal to the potential of the average density, as used in our van der Waals shift model. Likewise, averaging over local density fluctuations by rapid diffusion of the xenon and segmental motion of the polymer chain will tend to blur the distinctions between the two potentials; the average of the two potentials, weighted by their occurrence frequency, must reflect the average potential encountered by the xenon.

In the development of our model we assumed that the chain-bending modulus parameters were the same for all polymers studied here, and we therefore somewhat arbitrarily set $n$, the number of centers displaced by the diffusant, equal to $d / \lambda$, the diffusant diameter divided by the distance between centers on the chain. For these polymers this is approximately true, with the notable exception of PDMS. The chain-bending modulus for PDMS is 2 orders of magnitude smaller than for any of the other polymers studied, ${ }^{34}$ since the rotational barrier for the Si-O bond is only about $R T .^{62}$ Pace and Datyner ${ }^{33}$ calculate an effective value of $n$ from the chain-bending modulus, $\beta$ : for PE $(\beta=12 \mathrm{~kJ}-\mathrm{nm} / \mathrm{mol}) x$, the length of polymer chain disrupted by the xenon, is $3.2 \mathrm{~nm}$, giving a value of $n$ of ca. $24 \lambda$; for PDMS $(\beta=0.02 \mathrm{~kJ}-\mathrm{nm} / \mathrm{mol})$ $x$ is $0.56 \mathrm{~nm}$, making $n$ ca. $4 \lambda$. This difference in $n$ for PE and PDMS suggests that some effect of chain flexibility should exist. We note below however several aspects of the van der Waals shift model and data which question the applicability of chain flexibility to the calculation of ${ }^{129} \mathrm{Xe}$ van der Waals shifts.

A specific aim of the diffusion model is to calculate the energy barrier to diffusion, $E_{\mathrm{d}}$, which is a measure of the total energy required to create the hole for the diffusant. The ${ }^{129} \mathrm{Xe}$ van der Waals shift, on the other hand, is a measure of the difference in the energy of the polymer chain configuration with and without the xenon atom. In calculating $E_{\mathrm{d}}$ Pace and Datyner ${ }^{33}$ account for the energy needed to separate adjacent polymer chains due to the breaking of van der Waals bonds by insertion of the diffusant. Additionally, the energy needed to overcome bond rotation barriers for chain separation is accounted a
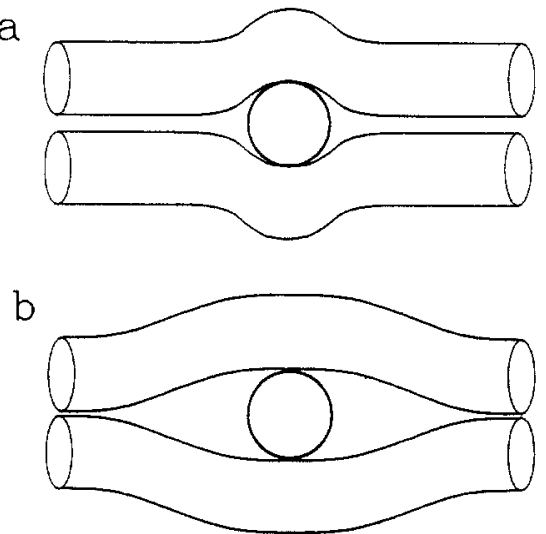

Figure 6. Schematic drawing of the effects of chain flexibility on polymer configuration in the presence of a diffusant molecule according to the model of Pace and Datyner: ${ }^{33}$ (a) flexible chain; (b) stiff chain.

for by the chain-bending modulus. Pace and Datyner ${ }^{33}$ make the assumption that only a small rotation occurs about each backbone bond, the rotation angle being a function of the chain-bending modulus. A stiffer chain therefore requires more backbone centers (more bonds) to participate in creating the hole for the diffusant, as indicated by the calculated values of $n$ for PE and PDMS and shown schematically in Figure 6. (DiBenedetto ${ }^{32}$ arrives at a similar result through his calculation of the number of centers over which the symmetric separation of chains occurs.) In such a view $\sigma_{w}$ would be proportional to $E_{\mathrm{d}}$. This is clearly not the case; the values of $E_{\mathrm{d}}$ for polyethylene and poly(dimethylsiloxane) differ by ca. a factor of 3,33 while their values of $\sigma_{\mathrm{w}}$ at $273 \mathrm{~K}$ (Figure 5) differ by only a few percent.

We present an alternative view: that the backbone bonds can undergo large bond angle distortions or interchange trans-gauche conformations. The polymer chain configuration in the presence of a diffusant molecule would be essentially the same as depicted in Figure 6 for a flexible chain regardless of chain stiffness. In this view $E_{\mathrm{d}}$ would reflect the energy due to the breaking of van der Waals bond as before. It would also include the energy barriers to trans-gauche interchange as well as the energy due to bond rotations which do not induce a trans-gauche conformational interchange. As in the previous case, $\sigma_{\mathrm{w}}$ would be a measure only of the difference in energy (van der Waals potential) between the two conformations of the polymer backbone. In this case the difference between $E_{\mathrm{d}}$ and $\sigma_{\mathrm{w}}$ is due primarily to the energy barriers to transgauche interchange. Thus we expect $\sigma_{\mathrm{w}}$ to reflect energies less than $E_{\mathrm{d}}$, with the exception of PDMS, where the energies should be similar since the energy barrier to transgauche interchange is essentially zero. Qualitatively, this view is supported by the ${ }^{129} \mathrm{Xe}$ NMR data. Based on our value of $0.012 \mathrm{~J}^{-1}$ for $K$, we use eq 9 to calculate van der Waals shift activation energies, $\Delta E_{\mathrm{w}}$, similar to the apparent activation energies for diffusion obtained from the Pace and Datyner model, ${ }^{34}$ from the ${ }^{129} \mathrm{Xe}$ chemical shifts. The values of $\Delta E_{\mathrm{w}}$ are given in Table III. They all fall within a relatively narrow range of $20-26 \mathrm{~kJ}$ and all are similar to the value of $24 \mathrm{~kJ}$ for PDMS. The values of $\Delta E_{\mathrm{w}}$ are less than the corresponding values for $E_{\mathrm{d}}$ (Table III), as expected, with the exception of PDMS, where $\Delta E_{\mathrm{w}}$ is greater than $E_{\mathrm{d}}(14 \mathrm{~kJ})$.

The discrepancy between $\Delta E_{\mathrm{w}}$ and $E_{\mathrm{d}}$ for PDMS is probably due, at least in part, to our assumption of the value of $n$, the number of polymer centers displaced by the diffusant. We had set $n$ equal to $d / \lambda$. We note that $\lambda$ is the average distance between centers along the polymer 
Table III. Values of $\Delta E_{\pi}$ and $E_{d}$

\begin{tabular}{lcc}
\hline & $\Delta E_{\mathrm{w}}(\mathrm{kJ} / \mathrm{mol})^{a}$ & $E_{\mathrm{d}}(\mathrm{kJ} / \mathbf{m o l})^{b}$ \\
\hline PIP & 22 & 40 \\
PVE & 26 & \\
PC & 23 & \\
PBD & 23 & \\
PIB & 26 & 14 \\
PDMS & 24 & 47 \\
PE & 22 & 48 \\
PEMA $^{c}$ & 22 & 48
\end{tabular}

a $\Delta E_{\mathrm{w}}$ from the measured resonance shifts extrapolated to $273 \mathrm{~K}$. ${ }^{b}$ From refs 33 and 34. ${ }^{c}$ PEMA above $T_{\mathrm{g}}{ }^{d}$ PEMA below $T_{\mathrm{g}}$.

chain, accounting for bond lengths, bond angles, and torsion angles along a straight chain. In bending around a diffusant molecule, the distance between centers may be altered, and in particular lengthened, by simple changes in the torsion angle. In this way the number of centers displaced by the diffusant can be minimized, leading to a decrease in $n$. (We note, for example, that the average $\mathrm{C}-\mathrm{C}$ bond length in PIP is some $50 \%$ longer than $\lambda$.) A decrease in the value of $n$ would lead us to choose a larger value for $K$, which in turn would decrease $\Delta E_{\mathrm{w}}$.

\section{Conclusions}

We have presented a van der Waals shift model of the ${ }^{129} \mathrm{Xe}$ NMR resonance shifts for xenon dissolved in polymers. Only one adjustable parameter, which is independent of the polymer, is necessary. The model accurately reflects the temperature dependence of the ${ }^{129} \mathrm{Xe}$ chemical shifts in polymers above $T_{\mathrm{g}}$. The predictions of the magnitudes of the shifts are somewhat less accurate. The deviations are probably due to small errors in the polymer parameters used, although variations in polymer chain flexibility or our assumption about the xenon's interaction with the free volume cannot be ruled out as sources of error.

In the van der Waals shift model presented here, the xenon creates a hole in the polymer and thereby increases the interchain separation by the difference between the xenon diameter and the free volume about the chains. Thus the ${ }^{129} \mathrm{Xe}$ chemical shift is sensitive to the free volume in the polymer. It should be noted however that, over the temperature ranges studied, the average local free volume is always less than the xenon volume so that the presence of the xenon distorts the local chain packing. In fact, the van der Waals shift model predicts that when the local free volume is larger than the xenon volume, the ${ }^{129} \mathrm{Xe}$ van der Waals shift will be zero. This is incorrect since adsorption of xenon onto the "wall" of the free volume cavity, implicit when the cavity is smaller than the xenon volume, is predicted to lead to a shift in the resonance frequency as well. ${ }^{30}$

We have shown a reasonable agreement between the van der Waals shift model's predictions and data from a suite of polymers. If one accepts the applicability of such a model, it is then possible to extract certain polymer parameters from the temperature dependence of the ${ }^{129} \mathrm{Xe}$ chemical shift. In particular, the van der Waals chain diameter in the low-temperature limit and the solubility parameter can be obtained given the density, volume thermal expansion coefficient, and the distance between chain centers, as we demonstrated above. The van der Waals chain diameter can be used to estimate the free volume of the system. The solubility parameter from fitting the NMR data should reflect only dispersion contributions, even in polar systems where dipolar effects also contribute. No values of the dispersive component of the solubility parameter for any polymers studied here are available to the best of our knowledge. We note, however, that the value of the solubility parameter obtained by fitting the model to the NMR data for PEMA falls in the low end of the range found in the literature, consistent with only the dispersive component contributing to the van der Waals shift.

Our van der Waals shift model of ${ }^{129} \mathrm{Xe}$ resonance shifts in polymers accounts for the effects of chain flexibility differently than the diffusion model on which it was based. From our qualitative assessment above, we conclude that chain flexibility has a minimal effect on the xenon resonance shift, in contrast to its much stronger effect on diffusion. This suggests that ${ }^{129} \mathrm{Xe}$ NMR can be used to independently check the parameters used in the diffusion model. It would be interesting to test this hypothesis by studying polymers with stiffer backbones than those in the present study, including liquid crystal forming polymers and possibly poly(vinyl chloride).

We are unable to determine the number of polymer backbone centers, $n$, displaced by the xenon atom. This is of little consequence in calculating the van der Waals shift, any error in $n$ being compensated by our choice of adjustable parameter $K$; however, it introduces an uncertainty in the values of the potentials associated with the two sites identified in our van der Waals shift model. We therefore assume the two sites are populated based on their frequency of occurrence and not the potential associated with each site. We interpret the success of this assumption to indicate that the average site in a real amorphous polymer is closely approximated by the average of the two sites in our model.

The subject of van der Waals shifts in liquids has attracted a lot of attention over the years. A number of models have been proposed to explain these shifts. In one of the most predictive of these models, ${ }^{20}$ linear correlations are found between experiment and theory for structurally related solvents such as linear alkanes or cyclic alkanes, but the correlations are poor among unrelated solvents. ${ }^{11}$ A model which correlates the van der Waals shifts of a wider variety of solvents would improve the understanding of resonance shifts in liquids. The van der Waals shift model presented here is devised for locally parallel linear molecules with essentially infinite length. The assumption that the molecules are locally parallel is important in the application of the model to diffusion; however, it is not clear that this assumption is required for application to NMR resonance shifts. Cell models have been applied to the properties of liquids, so it seems reasonable that a similar van der Waals shift model could be devised for liquids. We are currently investigating this possibility.

Acknowledgment. J.H.W. acknowledges the National Research Council and the Naval Research Laboratory for an NRC/NRL postdoctoral associateship. This work was partially supported by the Office of Naval Research. Helpful conversations with A. N. Garroway and J. P. Yesinowski are gratefully acknowledged. J.B.M. thanks Professor T. R. Stengle for the data on poly(ethyl methacrylate).

\section{References and Notes}

(1) NRC/NRL Post-Doctoral Associate. Present address: Chemistry Department, B-164, Florida State University, Tallahassee, FL 32306 .

(2) For a recent review, see: Dybowski, C.; Bansal, N.; Duncan, T. M. Annu. Rev. Phys. Chem. 1991, 42, 433.

(3) Ripmeester, J. A.; Davidson, D. W. J. Mol. Struct. 1981, 75, 67.

(4) Ripmeester, J. A. J. Am. Chem. Soc. 1982, 104, 209. 
(5) Brownstein, S. K.; Roovers, J. E. L.; Worsfold, D. J. Magn. Reson. Chem. 1988, 26, 392.

(6) Sefcik, M. D.; Schaefer, J.; Desá, J. A. E.; Yelon, W. B. Polym. Prepr. (Am. Chem. Soc., Div. Polym. Chem.) 1983, 24, 85.

(7) Stengle, T.R.; Williamson, K. L. Macromolecules 1987,20, 1428.

(8) Kennedy, G. J. Polym. Bull. 1990, 23, 605.

(9) Kentgens, A. P. M.; van Boxtel, H. A.; Verweel, R.-J.; Veeman, W. S. Macromolecules 1991, 24, 3712.

(10) Walton, J. H.; Miller, J. B.; Roland, C. M. J. Polym. Sci., Part B: Polym. Phys. 1992, 30, 527.

(11) Stengle, T. R.; Reo, N. V.; Williamson, K. L. J. Phys. Chem. $1981,85,3772$.

(12) Miller, K. W.; Reo, N. V.; Uiterkamp, A. J. M. S.; Stengle, D. P.; Stengle, T.R.; Williamson, K. L.Proc. Natl.Acad.Sci.U.S.A. $1981,78,4946$.

(13) For a review, see: Rummens, F. H. A. NMR Basic Principles and Progress; Springer-Verlag: New York, 1975.

(14) Rummens, F. H. A. Can. J. Chem. 1976, 54, 254.

(15) Rummens, F. H. A.; Mourits, F. M. Can. J. Chem. 1977, 55, 3021.

(16) Raynes, W. T.; Buckingham, A. D.; Bernstein, H. J. J. Chem. Phys. 1962, 36, 3481

(17) Rummens, F. H. A. J. Phys. Chem. 1968, 72, 2111.

(18) Linder, B. J. Chem. Phys. 1960, 33, 668.

(19) Howard, B. B.; Linder, B.; Emerson, M. T. J. Chem. Phys. 1962, 36,485 .

(20) Rummens, F. H. A. Chem. Phys. Lett. 1975, 31, 596.

(21) Rummens, F. H. A. J. Chim. Phys. 1975, 72, 448.

(22) Onsager, L. J. Am. Chem. Soc. 1936, 58, 1486.

(23) Stephen, M. J. Mol. Phys. 1958, 1, 223.

(24) Heckman, G.; Fluck, E. Mol. Phys. 1972, 23, 175.

(25) Geet, A. L. V. Anal. Chem. 1970, 42, 679.

(26) Geet, A. L. V. Anal. Chem. 1968, 40, 227.

(27) Yen, W. M.; Norberg, R. E. Phys. Rev. 1963, 131, 269.

(28) Brinkman, D.; Carr, H. Y. Phys. Rev. 1966, 150, 174.

(29) Kanegsberg, E.; Pass, B.; Carr, H. Y. Phys. Rev. Lett. 1969, 23, 572.
(30) Cheung, T. T. P.; Chu, P. J. J. Chem. Phys, 1992, 96, 9551.

(31) DiBenedetto, A. T. J. Polym. Sci., Part A 1963, 1, 3459.

(32) DiBenedetto, A. T. J. Polym. Sci., Part A 1963, 1, 3477.

(33) Pace, R. J.; Datyner, A. J. Polym. Sci., Polym. Phys. Ed. 1979, $17,437$.

(34) Pace, R. J.; Datyner, A. J. Polym. Sci., Polym. Phys. Ed. 1979, $17,453$.

(35) Pace, R. J; Datyner, A. J. Polym. Sci., Polym. Phys. Ed. 1979, $17,465$.

(36) Flory, P. J. Statistical Mechanics of Chain Molecules; Hanser: New York, 1989

(37) Burgess, W.H.;Hoppenberg, H. B.; Stannett, V.T. J. Macromol. Sci., Phys. 1971, B5, 23.

(38) Malhotra, B. D.; Pethrick, R. A. Eur. Polym. J. 1983, 18, 457.

(39) Malhotra, B. D.; Pethrick, R. A. Polym. Commun. 1983, 24, 165.

(40) Curro, J. J.; Roe, R.-J. Polymer 1984, $25,1424$.

(41) Victor, J. G.; Torkelson, J. M. Macromolecules 1987, $20,2241$.

(42) Lundstrom, J. E.; Bearman, R. J. J. Polym. Sci., Polym. Phys. Ed. 1974, 12, 97.

(43) Jameson, A. K.; Jameson, C. J.; Gutowski, H. S. J. Chem. Phys. $1970,53,2310$.

(44) Ferry, J. D. Viscoelastic Properties of Polymers; Wiley: New York, 1980.

(45) Roland, C. M. Macromolecules 1987, 20, 2557.

(46) Brandrup, J.; Immergut, E. H. Polymer Handbook; Wiley: New York, 1975.

(47) Roovers, J.; Toporowski, P. M. Macromolecules 1992, 25, 3454.

(48) Nagode, J. B.; Roland, C. M. Polymer 1991, 32, 505.

(49) Cohen, R. E. Elastomers and Rubber Elasticity; ACS Symposium Series 193; American Chemical Society: Washington, DC, $1982 ; \mathrm{p} 489$.

(50) Walton, J. H.; Miller, J. B.; Roland, C. M.; Nagode, J. B. Macromolecules, in press.

(51) Brandt, W. Phys. Rev. 1955, 98, 243.

(52) Scott, D. W.; Messerly, J. F.; Todd, S. S.; Guthrie, G. B.; Hossenlopp, I. A.; Moore, R. T.; Osborn, A.; Berg, W. T.; McCullough, J. P. J. Phys. Chem. 1961, 65, 1320. 